\title{
Role of uric acid and other parameters in sudden sensorineural hearing loss
}

\author{
Meltem Ilancioglu ${ }^{1} \cdot$ Ahmet Ural $^{2} \cdot$ Bengu Cobanoglu $^{3}{ }^{(D)} \cdot$ Asim Orem $^{4}$ \\ ${ }^{1}$ Department of Otorhinolaryngology, Aksaray State Hospital, Aksaray, Turkey \\ ${ }^{2}$ Department of Otorhinolaryngology, Bolu Abant Izzet Baysal University, Medical School, Bolu, Turkey \\ ${ }^{3}$ Department of Otorhinolaryngology, Karadeniz Technical University, Medical School, Trabzon, Turkey \\ ${ }^{4}$ Department of Biochemistry Karadeniz Technical University, Medical School, Trabzon, Turkey
}

\section{ABSTRACT}

Aim: To investigate the levels of metabolites (predominantly uric acid) effective on biochemical, and coagulation parameters and evaluate their effects on the onset, and course of the disease.

Methods: In this retrospective study, files of 92 patients hospitalized between January 2007, and December 2013, in our clinic with established diagnosis of sudden hearing loss were screened. The biochemical (predominantly uric acid), and hematological parameters were compared with those of the control group. In addition, the patient group was divided into two groups according to uric acid levels and the difference between the groups was investigated in terms of the onset or course of the disease.

Results: A significant difference was not detected between the patient, and the control groups regarding mean uric acid levels. Among biochemical parameters glucose, creatinine, and international normalized ratio (INR) were significantly higher $(p<0.05)$ while a significant intergroup difference was not detected as for other parameters. A significant intergroup difference was not detected in mean pure- tone averages, and mean hearing gain at admission between two groups formed based on uric acid levels, while post-treatment pure-tone average was significantly better in patients with higher serum uric acid levels. In the patient group, uric acid levels were significantly higher in patients with partial hearing loss relative to those with total loss.

Conclusion: In our study, we could not find a significant difference between the patient and the control groups as for uric acid levels. However, we have encountered evidence supporting the possible role of serum uric acid levels in the prognosis of sudden hearing loss.

Keywords: Sudden hearing loss, uric acid, oxidative stress, vascular injury.

(C) 2020 experimentalbiomedicalresearch.com

Dr. Bengu Cobanoglu,

Department of Otorhinolaryngology, Karadeniz

Technical University, Medical School, Trabzon, Turkey

E-mail: benguyc@gmail.com

Received: 2019-12-03 / Accepted: 2020-01-23

Publication Date: 2020-03-06

\section{Introduction}

"Sudden Hearing Loss" (SHL) is defined as development of sensorineural hearing loss (SNHL) of at least $30 \mathrm{~dB}$ at three contiguous frequencies within less than 72 hours [1]. Although its etiology has not been clarified definitively, vascular theory is the most 
accepted one [2]. Cochlear perfusion with terminal arteries, and its higher sensitivity to hypoxia tend to support vascular injury as an etiologic factor. Available evidence indicates that metabolic diseases with microvascular effects such as diabetes, and hyperlipidemia are effective in the development of SHL [3]. Still, SHL secondary to neurological damage due to viral, neurotoxic, traumatic etiologies can be also seen [1].

Uric acid is the end-product of purine metabolism, and its levels increase in line with oxidative stress. In various studies performed, increased levels of uric acid have been indicated in conditions developed in association with vascular pathologies as myocardial infarction, and cerebrovascular events, and also its close association with endothelial dysfunction has been reported [4]. However in a few studies performed in recent years, correlations between lower uric acid levels, and increasing prevalence, and deteriorated course of some neurological diseases as Parkinson's disease, and Alzheimer's disease have been detected [5].

This trial aims to investigate the role of uric acid in the etiology, and prognosis of SHL. Together with uric acid, other metabolites and blood level parameters that might affect plasma have been analyzed.

\section{Materials and Methods}

The study was performed after obtaining approval of the ethics committee of the faculty (Decision \#17522305/678). File numbers of the inpatients treated in our ENT service between January 2007 and December 2013 were acquired from informatics department of otorhinolaryngology department of our tertiary care center. The cases coded as "Sudden idiopathic hearing loss" according to International Classification of Diseases (ICD) were taken into account. Consequently, medical files of 56 female, and 82 male patients who were hospitalized, and treated with the diagnosis of sudden hearing loss were obtained. Fifteen female and 12 male patients whose laboratory data at admission could not be obtained were excluded from the study. Among the remaining cases, one female patient with chronic kidney disease, 2 patients (1 F and $1 \mathrm{M}$ ) diagnosed as schwannoma on MRI, and 2 female patients with presumed diagnosis of fistula were not included in the study. Besides 14 patients with diabetes (12 M and $2 \mathrm{~F}$ ) were also excluded from the study. As a result, a total of 92 patients ( $35 \mathrm{~F}$ and $57 \mathrm{M}$ ) were included in the study (Table 1).

Table 1. Distribution of the patients included in, and excluded from the study based on causative factors.

\begin{tabular}{|l|l|l|l|}
\hline Parameters & Female & Male & Total \\
\hline $\begin{array}{l}\text { Patients with the } \\
\text { diagnosis of sudden } \\
\text { hearing loss }\end{array}$ & 56 & 82 & 138 \\
\hline $\begin{array}{l}\text { Laboratory data at } \\
\text { admission not obtained }\end{array}$ & 15 & 12 & 27 \\
\hline Chronic kidney disease & 1 & 0 & 1 \\
\hline Schwannoma & 1 & 1 & 2 \\
\hline Fistula & 2 & 0 & 2 \\
\hline $\begin{array}{l}\text { Diabetes and } \\
\text { hypertension }\end{array}$ & 2 & 12 & 14 \\
\hline Included in the study & 35 & 57 & 92 \\
\hline
\end{tabular}

Ninety-two (35 F and $57 \mathrm{M}$ ) patients with similar age, and gender-distribution relative to the patient group who applied to our outpatient clinic within the first 3 months of the year 2014 without any degree of hearing loss, and chronic disease and also underwent complete routine biochemical tests constituted the control group. In this study the following biochemical, and hematological parameters were evaluated: uric 
acid, FBG, blood urea nitrogen (BUN), creatinine, hemoglobin $(\mathrm{Hb})$, platelet, mean platelet volume (MPV), platelet distribution width (PDW), platelet count or plateletcrit (PTC), prothrombin time (PT), partial thromboplastin time (PTT), and international normalized ratio (INR). These hematologic parameters of the patient, and the control groups were compared, and any significant intergroup difference (if any) was evaluated.

Average, and median uric acid values were 4.6 $\mathrm{mg} / \mathrm{dl}$, and $4.5 \mathrm{mg} / \mathrm{dl}$, respectively. Based on the median uric acid value, the patients were divided into 2 groups as those with uric acid levels of $\leq 4.5 \mathrm{mg} / \mathrm{dL}$ ( $\mathrm{n}=47$ ), and $>45 \mathrm{mg} / \mathrm{dL}$ $(n=45)$. Pure-tone averages at the onset of the disease, and after the treatment, and also mean hearing gains of these groups were compared. In groups of patients formed based on the degree of the hearing loss, and audiometric pattern at admission, any significant difference (if any) as for mean uric acid levels, and a difference (if any) in the intragroup distributions of the groups constructed based on uric acid levels, and hearing gains were investigated. The patients were divided into 2 groups based on pure-tone averages at admission as partial $(<90 \mathrm{~dB})$, and total hearing loss ( $\geq 90 \mathrm{~dB}$ ). Based on the median value, the patients were divided into 2 groups as those with uric acid levels of $\leq 4.5 \mathrm{mg} / \mathrm{dL}(\mathrm{n}=47)$, and $>45 \mathrm{mg} / \mathrm{dL}(\mathrm{n}=45)$. Pure-tone averages at the onset of the disease, and after the treatment, and also mean hearing gains of these groups were compared. In groups of patients formed based on the degree of the hearing loss, types of audiograms recorded at admission, any significant difference (if any) as for mean uric acid levels, and a difference (if any) in the intragroup distributions of the groups constructed based on uric acid levels, and hearing gains were investigated.
The patients were divided into 2 groups based on pure-tone averages at admission as partial $(<90 \mathrm{~dB})$, and total hearing loss $(\geq 90 \mathrm{~dB})$. When categorized in types of audiograms, upward-sloping, and flat-type audiograms which have been associated with better prognosis in literature studies [1] were included in Group 1, while flat type audiograms, and those indicating total hearing loss were categorized in Group 2.

Treatment response was evaluated using Siegel classification [6], and the patients were divided into groups based on baseline pure tone averages as calculated in post-treatment audiometric tests as follows: hearing gain $>15$ $\mathrm{dB}$ (no improvement); $\geq 15 \mathrm{~dB}$, and mean hearing acuity $>25 \mathrm{~dB}$ (partial improvement) pure-tone average in audiometry was $\geq 25 \mathrm{~dB}$ (total recovery).

\section{Statistical analysis}

For statistical analysis SPSS (Statistical Package for Social Sciences) for Windows 13.0 program was used. In the comparison of data of the patient, and the control groups which demonstrated normal distribution as age, FBG, uric acid, platelet counts, MPV, and PTT values, Student $-\mathrm{T}$ test was used. For the comparison of variables with non-normal distribution such as BUN, creatinine, hemoglobin, platelet, PDW, INR, and PT values, Mann -Whitney U (MWU) test was employed.

\section{Results}

\section{Demographic and biochemical comparisons}

Mean age of 35 female, and 57 male patients enrolled in the study was $43.6 \pm 16.84$ (10-79) years. Mean age of the control group consisting of equal number of male and female patients was $42.8 \pm 16.5$ (19-90) years. Any significant 
intergroup difference was not detected regarding mean age of the patients $(\mathrm{p}=0.525)$.

Mean uric acid levels of the patient, and control groups were $4.65(\mathrm{n}=92)$, and $4.63 \mathrm{mg} / \mathrm{dL}$ $(n=92)$, respectively without any statistically significant intergroup difference $(\mathrm{p}=0.871)$. Mean uric acid levels of the female study participants in the patient, and the control groups were $4.00(\mathrm{n}=36)$, and $3.789 \mathrm{mg} / \mathrm{dL}$ $(n=36)$, respectively without any statistically significant difference $(\mathrm{p}=0.270)$. Mean uric acid levels of the male study participants of the patient, and the control groups were $5.06 \mathrm{mg} / \mathrm{dL}$ $(\mathrm{n}=56)$, and $5.16 \mathrm{mg} / \mathrm{dL}$, respectively $(\mathrm{n}=56)$ without statistically significant intergroup difference $(\mathrm{p}=0,619)$.

Among other parameters studied, creatinine, glucose, INR, and PT values were significantly higher in the patient group. BUN, Hb, platelet, MPV, plateletcrit, PDW, and PTT were not statistically significantly different between both groups. (Table 2)

\section{Demographic and biochemical data of the patient group}

Mean, and median uric acid levels of the patient group were 4.65 , and $4.5 \mathrm{mg} / \mathrm{dl}$, respectively. The patients were divided into two groups based on median uric acid value as Group 1 $(\mathrm{n}=47$; uric acid $\leq 4.5 \mathrm{mg} / \mathrm{dL})$ and Group 2, $(\mathrm{n}=45 ;$ uric acid $>4.5 \mathrm{mg} / \mathrm{dL})$. Among biochemical metabolites, and coagulation parameters $\mathrm{Hb}, \mathrm{BUN}$, and creatinine were significantly higher in Group $2(\mathrm{p}<0.01)$, while other parameters did not differ significantly between groups. These two groups were compared as for treatment onset, and prognosis. In Group 1, 46, and in Group 2, 38 patients had their audiometric records. Pure-tone averages of the study participants estimated at admission were compared, and any statistically significant difference could not be found between groups (Group 1, 83. $23 \mathrm{~dB}$, and Group 2, $74.11 \mathrm{~dB}$ ) $(\mathrm{p}=0.072)$. Post-treatment audiometric test records were available for 46 individuals in

Table 2. Comparison of laboratory parameters between the patient, and control groups.

\begin{tabular}{|l|l|l|l|l|l|}
\hline Parameters & Patient & Control & Range & $\boldsymbol{p}$ Value & Test \\
\hline Age (years) & 43,6 & 42,0 & - & 0,525 & St. T \\
\hline Uric acid (mg/d19 & 4,65 & 4,63 & $2,6-7,0$ & 0,871 & St. T \\
\hline BUN (mg/dl9 & 15,3 & 14,2 & $6-23$ & 0,079 & MWU \\
\hline Creatinine (mg/dl) & 0,76 & 0,75 & $0,5-1,2$ & $0,008 *$ & MWU \\
\hline Glucose (mg/dl) & 95,4 & 87,9 & $55-105$ & $<0,01 *$ & St. T \\
\hline Hemoglobin $(\mathrm{gr} / \mathrm{dl})$ & 13,9 & 14,0 & $12-17$ & 0,665 & MWU \\
\hline Platelet $\left(\mathrm{x} 10^{3 / \mu l}\right)$ & 261 & 260 & $130-400$ & 0,885 & St. T \\
\hline MPV (fl) & 8,3 & 8,48 & $7,4-11$ & 0,346 & St. T \\
\hline PLT $(\%)$ & 0,21 & 0,21 & $0-0,99$ & 0,298 & MWU \\
\hline PDW $(\%)$ & 16,7 & 16,7 & $0-99,9$ & 0,848 & MWU \\
\hline PTT (sec) & 28,1 & 29,05 & $22-40$ & 0,085 & St. T \\
\hline INR & 1,06 & 1,01 & - & $<0,01 *$ & MWU \\
\hline PT (sec) & 13,3 & 12,9 & $11-14$ & $0,013^{*}$ & MWU \\
\hline
\end{tabular}

BUN: blood urea nitrogen; MPV: mean platelet volüme; PDW: platelet distribution width; PTC: platelet count or plateletcrit; PT: prothrombin time; PTT: partial thromboplastin time; INR: international normalized. 
Group 1 and 36 participants in Group 2. Posttreatment pure-tone averages were 70.16 $\mathrm{dB}$ in Group 1, and $54.58 \mathrm{~dB}$ in Group 2, with a statistically significant intergroup difference $(\mathrm{p}=0.034)$. Whereas mean hearing gain in Groups 1, and 2 were 12.98, and $20.78 \mathrm{~dB}$, respectively, without any statistically significant intergroup difference $(\mathrm{p}=0.067)$. In summary, even a statistically significant difference could not be found, in the group with higher uric acid level milder hearing loss was detected at the onset of the disease with acquisition of higher hearing gain. Besides post treatment pure- tone averages were significantly better (Table 3). hearing loss were $4.8 \mathrm{mg} / \mathrm{dl}$, and $4.1 \mathrm{mg} / \mathrm{dl}$, respectively with a statistically significant intergroup difference $(\mathrm{p}=0.008)$. In other words as uric acid levels increased, onset of sudden hearing loss was less aggressive.

Audiometric records of 80 patients could be accessible. Patients with upward-sloping, and flat audiograms had a better prognosis (Group 1, $\mathrm{n}=46$ ) while patients with audiograms demonstrating a downward-sloping pattern and total loss were evaluated in Group $2(n=34)$. Mean uric acid value of the group with upwardsloping, and flat -type audiograms, and hence better prognosis was $4.7 \mathrm{mg} / \mathrm{dL}$, while that of the patients with poor prognosis, who had

Table 3. Comparison of admission, and post-treatment pure-tone average, and gain values of the patients grouped based on their uric acid levels expressed in decibels.

\begin{tabular}{|l|l|l|l|}
\hline \multirow{2}{*}{ Parameters } & \multicolumn{3}{|c|}{ Uric acid range $(\mathbf{m g} / \mathbf{d l})$} \\
\cline { 2 - 4 } & Uric acid $\leq \mathbf{4 , 5}(\mathrm{n}=\mathbf{4 7})$ & Uric acid $\mathbf{4 , 5}(\mathrm{n}=\mathbf{4 5})$ & $\boldsymbol{p}$ Value \\
\hline PTA at admission $(\mathrm{dB})$ & $83,23 \quad(\mathrm{n}=46)$ & $74,11 \quad(\mathrm{n}=38)$ & 0,072 \\
\hline Posttreatment PTA $(\mathrm{dB})$ & $70,26 \quad(\mathrm{n}=46)$ & $54,58 \quad(\mathrm{n}=36)$ & $0,034^{*}$ \\
\hline Hearing gain $(\mathrm{dB})$ & $12,98 \quad(\mathrm{n}=46)$ & $20,78 \quad(\mathrm{n}=36)$ & 0,067 \\
\hline
\end{tabular}

PTA: Pure-tone average.

Table 4. Distribution of patients grouped based on uric acid levels according to gain groups.

\begin{tabular}{|l|l|l|l|}
\hline \multirow{2}{*}{ Parameters } & \multicolumn{2}{|c|}{ Uric acid range (mg/dl) } \\
\cline { 2 - 4 } & Uric acid $\leq \mathbf{4 , 5}(\mathbf{n = 4 7 )}$ & Uric acid $>\mathbf{4 , 5}(\mathbf{n = 4 5})$ & Total \\
\hline No hearing gain & 33 & 18 & 51 \\
\hline Partial hearing gain & 9 & 10 & 19 \\
\hline Complete hearing gain & 4 & 8 & 12 \\
\hline Total & 46 & 36 & 82 \\
\hline
\end{tabular}

Hearing test records of 8 out of 92 patients at admission were not available, and the remaining 84 patients were divided into groups with partial, and total hearing loss (Group 1, $\mathrm{n}=53$, and Group 2, $\mathrm{n}=29$ ). Average uric acid levels of the patients with partial, and total downward-sloping, and total loss type audiograms was $4.3 \mathrm{mg} / \mathrm{dL}$, without any significant intergroup difference $(\mathrm{p}=0.149)$.

The patients were divided into three groups based on their hearing gains they retrieved from the treatment using modified Siegel 
classification [6]. The patients were divided into three groups. Mean hearing gain of the patients in Group 1 was less than $15 \mathrm{~dB}$ after treatment (no improvement) while of Group 2 was less than $25 \mathrm{~dB}$ after treatment, and their hearing acuity was still less than $25 \mathrm{~dB}$ (partial improvement). In Group 3 mean hearing gain was above $25 \mathrm{~dB}$ (total improvement group).

Posttreatment pure-tone averages of 82 out of 92 patients were available. These patients were in the groups of no hearing gain $(n=51 ; 62.2 \%)$, partial gain $(\mathrm{n}=19 ; 23.2 \%)$, and total gain $(n=12 ; 14.6 \%)$. When these groups were compared according to 2 groups divided based on uric acid values as $\leq 4.5 \mathrm{mg} / \mathrm{dL}$, and $>4.5$ $\mathrm{mg} / \mathrm{dL} \mathrm{p}$ was equal to 0.098 according to $c h i$ square test, and distribution of the groups were statistically significantly different. However, when distributions into groups were analyzed numerically, the number of patients with hearing gain was higher in the group with increased uric acid levels, while lesser number of patients in this group had not achieve any hearing gain from the treatment (Table 4).

\section{Discussion}

In approximately $80 \%$ of SHL patients an identifiable etiology is not found, and in its etiology mostly vascular theory has been emphasized [2]. Accordingly, hearing loss can arise from a sudden vascular bleeding in the cochlea [7], an occlusion caused by an embolus etc. [8], vasospasm [9] or alteration of blood viscosity [10].

In this study our target was essentially to analyze levels of uric acid whose role in vascular diseases, and oxidative stress have gained prominence in recent years in patients with SHL, compare its levels with those of the control group, and investigate its impact on prognosis. In our study, starting from vascular theory, a group of 92 patients with SHL were investigated as for uric acid levels, hematologic parameters which will effect blood viscosity, and coagulation, and biochemical variables which might exert an effect on plasma levels of uric acid, and these variables were compared with those of the control group

In a study performed in the year 2012, a group of 147 patients with SHL was compared with a group of 103 control subjects with respect to similar parameters, and any significant intergroup difference could not be found regarding BUN, and creatinine values [11]. In our study we also investigated uric acid values, renal functions, and hence creatinine, and BUN values which reflect uric acid clearance. Mean creatinine value in the patient group was 0.01 $\mathrm{mg} / \mathrm{dL}$ higher than that of the control group that was statistically significant. Mean creatinine value in the patient group was $0.01 \mathrm{mg} / \mathrm{dL}$ higher than that of the control group which was statistically significant. Though higher mean BUN, and uric acid values were measured in the patient group, a significant difference was not found relative to the control group.

In a study performed in 2014, all patients hospitalized with the diagnosis of SHL had undergone 75 gr oral glucose tolerance test, and more improved prognosis was detected in the normo-glycemic group relative to the group with impaired glucose tolerance test, and diabetes [12]. Even though diabetic patients were not included in our study, mean FBG level in the patient group was significantly higher when compared with the control group. These data may signify that though not at diabetic levels, higher fasting blood glucose (FBG) levels may be potentially effective factors in vascular etiopathogenesis of SHL.

Myeloprolipherative disorders as essential thrombocytosis, and polycythemia can predispose to vascular pathologies by increasing blood viscosity. In a study where 147 
SHL cases were compared as treatment responsive $(n=102)$, and refractory $(n=45)$ patients, hemoglobin levels were found to be significantly higher in the treatment-refractory group [11]. In our study, mean hemoglobin value in the control group was $0.1 \mathrm{mg} / \mathrm{dL}$ higher without any statistically significant intergroup difference.

Platelets are the smallest cells in the peripheral blood, and they are responsible from the release of mediators involving in coagulation, inflammation, thrombosis, and atherosclerosis [13]. In a study performed in the year 2006, any significant difference could not be found between the control, and SHL groups regarding PT, PTT, and platelet counts [14]. We did not come across a significant difference between the patient, and the control groups when compared in terms of platelet counts, and plateletcrit values. Although any difference was not found between both groups as for PTT, INR, and PT -albeit within their normal limitsthe levels of these parameters were significantly higher in the patient group, and higher levels of these parameters lead to a delay in coagulation with resultant increase in the risk of bleeding, and on the contrary a decrease in the risk of thrombus formation.

Mean platelet volume (MPV) is the average of all platelet volumes, and it is used as a biomarker in the evaluation of production, and function of platelets. Large platelets are more active both from metabolic, and enzymatic aspects. Hence when compared with small platelets, they have a higher tendency to precipitate, and so an increased coagulation potential. In cases with vascular occlusion, acute, and chronic syndromes, MPV levels increase, but decrease in infections, autoimmune diseases or inflammatory conditions. In our study any significant difference was not found between the patient, and the control groups as for MPV, and PDW values.

In studies performed in recent years the relationship between uric acid, and vascular injury of vascular, and renal tissues has been advocated. Pro-inflammatory, complement, platelet, and coagulation cascade activating, neutrophil, and macrophage stimulating, protease, and oxidant synthetizing effects of urate crystals are already known [4]. Deterioration of endothelial dysfunction has been detected in patients who were given uric acid preparations, and also an association between endogenous uric acid concentration and severity of endothelial dysfunction has been revealed [15]. Similarly, incidence rates of atherosclerosis, arteriosclerosis, glomerulosclerosis, vestibular ataxia, and renovascular pathologies increase in gout patients [16].

In the literature, higher uric acid levels have been reported to increase risks of coronary artery disease, and cerebrovascular disease. Increased uric acid levels have been thought to contribute to the atherosclerotic process by effecting endothelial functions, oxidative metabolism, adhesion, and aggregation of platelets [4]. As reported in the literature studies, after excluding other factors, each 1 $\mathrm{mg} / \mathrm{dL}$ increase in serum uric acid levels both in men, and women induces significant increases in cardiovascular, and coronary artery disease-related mortality rates [17]. Ding et al. advocated possible association between hyperuricemia, and thrombotic complications [18].

However, uric acid provides more than $50 \%$ of antioxidant capacity of the blood, and exerts stabilizing effects on vitamins $\mathrm{C}$, and $\mathrm{E}$. Therefore, potential increase in uric acid levels has been suggestively associated with an antioxidant response to oxidative stress. It has 
been thought that acute increase in uric acid levels is especially a response to oxidative stress, while its chronic increase is thought to be a risk factor for coronary, and cardiovascular diseases [19]. Even if its harmful pro-oxidant effect prevails over its beneficial antioxidant effect, its beneficial antioxidant effects seem to be more effective in the central nervous system [5]. In our study a significant difference was not detected in uric acid levels between the patient, and the control groups. In the literature we haven't encountered any study which investigated the relationship between uric acid, and SHL. In accessible literature studies the effects of uric acid, and other risk factors on SHL has been investigated. In a study by Friedrich et al. the frequency of vascular risk factors as hyperuricemia, hyperglycemia, and cigarette smoking in 264 patients with SHL had been investigated, and the authors had detected higher rates of hyperuricemia, and hyperglycemia in patients with SHL relative to healthy population. In the same study, an inverse correlation was detected between recovery of hearing function, and number of risk factors [20]. In another study 163 patients with SHL were analyzed as for risk factors including hypertension, hyperlipidemia, smoking, hyperuricemia, and obesity, and significantly greater number of vascular risk factors were detected in the patient group relative to the control group [21].

In our study we evaluated the impact of uric acid levels on the onset, and prognosis of SHL, and 91 of 92 patients had uric acid levels within normal range, and only one patient had an uric acid level above $7.5 \mathrm{mg} / \mathrm{dL}(8,4 \mathrm{mg} / \mathrm{dl})$. This patient applied with a hearing loss of $70 \mathrm{~dB}$, and did not recover at all after treatment When the patient population were divided into groups with median uric acid levels of $\leq 4.5 \mathrm{mg} / \mathrm{dl}$, and $>4.5 \mathrm{mg} / \mathrm{dl}$, and pure-tone averages at admission were compared, pure-tone averages were found to be $83.23 \mathrm{~dB}$, and $74.11 \mathrm{~dB}$ in Groups 1, and 2, respectively without any statistically significant intergroup difference ( $\mathrm{p}=0.072$ ). Posttreatment pure-tone averages of Groups 1, and 2 were 70.26, and $54.58 \mathrm{~dB}$, respectively with a statistically significant intergroup difference $(\mathrm{p}=0.034)$. Hearing gains of Groups 1, and 2 were 12.98, and $20.78 \mathrm{~dB}$ without any statistically significant intergroup difference $(\mathrm{p}=0.067)$. In conclusion, although a statistically significant intergroup difference was not found, in the group with higher uric acid level at the onset of the disease hearing loss was less severe, and higher hearing gain was obtained.

The patient population was divided into groups with partial, and total hearing loss (Group 1, $\mathrm{n}=53$, and Group 2, $\mathrm{n}=29$ ) with median serum uric acid levels as 4.8, and $4.1 \mathrm{mg} / \mathrm{dL}$, respectively with a statistically significant intergroup difference $(p=0.008)$. Similarly, the patients with better prognosis, and upwardsloping and flat-type audiograms $(n=46)$, and those with downward- sloping type , and total hearing loss $(n=34)$ were compared as for mean serum uric acid levels (Group 1, $4.7 \mathrm{mg} / \mathrm{dL}$, and Group 2, $4.3 \mathrm{mg} / \mathrm{dL}$ ) without any significant intergroup difference $(p=0.149)$. In other words, onset of sudden hearing loss was less aggressive as serum uric acid levels increased.

Distribution of two groups constructed based on uric acid levels was separated into 3 groups as for hearing gains, and still any significant difference was not detected between these two groups $(\mathrm{p}=0.098)$. Though any intergroup difference was not detected, when distribution of patients was examined greater number of patients were seen in the group with lower uric acid levels, while in the group with higher uric acid levels the patients with partial, and complete recovery were more numerous. 
As we already mentioned, most of the studies cited in the literature, have correlated uric acid levels, and sudden vascular diseases, worse disease prognosis, and thrombosis. However, in some studies deteriorated course of some neurological diseases such as multiple sclerosis [22], Parkinson's disease [23], Alzheimer's disease [24] in patients with lower uric acid levels have been reported [5]. Based on our data, the group with higher uric acid levels had better pre-and post-treatment pure-tone averages, hence treatment gain, and improved prognosis. Starting from the hypothesis that acute increase in uric acid levels occurs in response to oxidative stress, and uric acid ameliorates neuronal damage thanks to its antioxidant, and immunoregulatory effects [5, 19] increased uric acid level can be suggested as a positive prognostic factor in SHL. In our study, scarce number of patients with supranormal uric acid levels, lack of any prominent difference between groups determined based on median uric acid value, unequal number of male, and female patients in groups precluded accurate interpretation of the results. In the years to come, if a larger-scale patient population with uric acid levels higher than normal range can be investigated, then it will be possible to achieve more accurate results.

This is a retrospective study whose data set was constructed by screening the files of patients with SHL who applied to our clinic within the previous 6 years. Laboratory values of the patients with SHL were derived from the measurements made within 5 years, and for the control group, data were harvested among the measurements made for the patients applied for preoperative preparation for anesthesia within the previous year. Since medical files were screened to obtain data about symptoms, use of medication, and audiogram results, and physicians who prepared the files changed during 5 years, some omissions may be found in patient information. In the future, suspicious data stemming from various factors including concomitant diseases, and timing of laboratory tests can be minimized by designing prospective studies.

\section{Conclusion}

In conclusion, in this study we evaluated uric acid that is thought to be effective in endothelial dysfunction, at the onset, and progression of SHL, but we couldn't find a significant difference between the patient, and the control groups as for uric acid levels. However, a significant difference was found between the patient, and the control groups with respect to other hematological, and biochemical parameters including creatinine, INR, and PT. Besides, we found that supported better disease onset, and prognosis in patients with higher uric acid levels. In conclusion, well-planned prospective studies with larger sample size should be performed to obtain more accurate data about vascular etiology of the sudden hearing loss, and the role of other biochemical parameters in the etiopathogenesis of SHL.

Funding: There is no financial support and sponsorship

Conflict of Interest: The authors declare that they have no conflict of interest.

Ethical statement: The study was performed after obtaining approval of the ethics committee of the faculty (Decision \#17522305/678).

ORCID iD of the author(s)

Meltem Ilancioglu 10000-0001-7549-367X

Ahmet Ural 10000-0002-6088-1415

Bengu Cobanoglu /0000-0003-3701-1697

Asim Orem 10000-0001-8450-5783 


\section{References}

[1]Kuhn M, Heman-Ackah SE, Shaikh JA, et al. Sudden sensorineural hearing loss: a review of diagnosis, treatment, and prognosis. Trends Amplif. 2011; 15(3):91105.

[2]Byl FM Jr. Sudden hearing loss: eight years' experience and suggested prognostic table. Laryngoscope. 1984; 94(5 Pt 1):64761.

[3]Kakarlapudi V, Sawyer R, Staecker H. The effect of diabetes on sensorineural hearing loss. Otol Neurotol. 2003; 24(3):382-86.

[4]Tavil Y, Kaya MG, Oktar SO, et al. Uric acid level and its association with carotid intima media thickness in patients with hypertension. Atherosclerosis. 2008:197(1):159-16.

[5]Alvarez-Lario B, Macarrón-Vicente J. Is there anything good in uric acid? QJM. 2011; 104(12):1015-1024.

[6]Siegel LG. The treatment of idiopathic sudden sensorineural hearing loss. Otolaryngol Clin North Am. 1975:8(2):46773.

[7]Colclasure JB, Graham SS. Intracranial aneurysm occurring as sensorineural hearing loss. Otolaryngol Head Neck Surg. 1981; 89(2):283-87.

[8]Jaffe BF. Sudden deafness--a local manifestation of systemic disorders: fat emboli, hypercoagulation and infections. Laryngoscope. 1970; 80(5):788801.

[9]Mattox DE, Lyles CA. Idiopathic sudden sensorineural hearing loss. Am J Otol. 1989; 10(3):242-47.

[10] Capaccio P, Ottaviani F, Cuccarini V, et al. Genetic and acquired prothrombotic risk factors and sudden hearing loss. Laryngoscope. 2007; 117(3):547-51.
[11] Yasan H, Tüz M, Yariktaş M, et al. The significance of routine laboratory parameters in patients with sudden sensorineural hearing loss. Indian $\mathbf{J}$ Otolaryngol Head Neck Surg. 2013;65(Suppl 3):553-56

[12]Ryu OH, Choi MG, Park $\mathrm{CH}$, et al. Hyperglycemia as a potential prognostic factor of idiopathic sudden sensorineural hearing loss. Otolaryngol Head Neck Surg. 2014; 150(5):853-58.

[13] Karli R, Alacam H, Unal R, et al. Mean platelet volume: is it a predictive parameter in the diagnosis of sudden sensorineural hearing loss?. Indian J Otolaryngol Head Neck Surg. 2013; 65(4):350-53.

[14]Cadoni G, Agostino S, Scipione S, et al. Sudden sensorineural hearing loss: our experience in diagnosis, treatment, and outcome. J Otolaryngol. 2005; 34(6):395401.

[15] Waring WS, Webb DJ, Maxwell SR. Effect of local hyperuricemia on endothelial function in the forearm vascular bed. British J Clin Pharmacol. 2000:49(1):511.

[16] Kanellis J, Kang DH. Uric acid as a mediator of endothelial dysfunction, inflammation, and vascular disease. Semin Nephrol. 2005; 25(1):39-42.

[17]: Gonick HC, Rubini MD, Gleason IO, et al. The renal lesion in gout. Ann Intern Med. 1965; 62:667-74.

[18]Ding DD, Wang W, Cui ZG, et al. Changes of platelet $\alpha$-particle membrane protein, platelet activating factor and platelet parameters in patients with hyperuricemia. Zhongguo Shi Yan Xue Ye Xue Za Zhi. 2012; 20(2):394-97.

[19] de Oliveira EP, Burini RC. High plasma uric acid concentration: causes and consequences. Diabetol Metab Syndr. 2012; $4: 12$. 
[20] Schmolke B, Hörmann K. Vaskuläre Risikofaktoren beim Hörsturz und ihre Häufigkeit in der Normalbevölkerung. Eine retrospektive Studie [Vascular risk factors of sudden deafness and its incidence in the normal population. A retrospective study]. HNO. 1990; 38(12):440-45.

[21] Friedrich G. Zur Atiologie und Pathogenese des Hörsturzes [Etiology and pathogenesis of sudden deafness]. Laryngol Rhinol Otol (Stuttg). 1985; 64(2):62-66.

[22] Toncev G, Milicic B, Toncev S, et al. Serum uric acid levels in multiple sclerosis patients correlate with activity of disease and bloodbrain barrier dysfunction. Eur J Neurol. 2002; 9(3):221-26.

[23] Gao X, Chen H, Choi HK, et al. Diet, urate, and Parkinson's disease risk in men. Am J Epidemiol. 2008; 167(7):831-38.

[24] Kim TS, Pae CU, Yoon SJ, et al. Decreased plasma antioxidants in patients with Alzheimer's disease. Int J Geriatr Psychiatry. 2006; 21(4):344-48.

[25] Martinon F. Update on biology: uric acid and the activation of immune and inflammatory cells. Curr Rheumatol Rep. 2010; 12(2):135-41. 